Pacific Journal of Mathematics

HEREDITARY CROSSED PRODUCT ORDERS 


\section{HEREDITARY CROSSED PRODUCT ORDERS}

\section{H. MerkLeN}

In this paper one deals with crossed product orders $\Lambda$ of the following form: Let $\mathscr{R}$ be a Dedekind domain with quotient field $\mathscr{F}$ and $\mathscr{E}$ a semisimple, commutative, algebra of finite dimension over $\mathscr{F}$. Let $\mathscr{G}$ be a finite subgroup of the group of automorphisms of $\mathscr{E}$ whose fixed subalgebra is $\mathscr{F}$, and let $\Lambda_{0}$ be an $\mathscr{R}$-order in $\mathscr{E}$, which is $\mathscr{G}$-stable. Then, if [f] is an element of the second cohomology group $H^{2}\left(\mathscr{G}, U\left(\Lambda_{0}\right)\right)$, our order is $\Lambda=\Delta\left(\mathfrak{f}, \Lambda_{0}, \mathscr{G}\right)$. One is interested in the set of all maximal orders in $\mathscr{A}=\Delta(\mathfrak{f}, \mathscr{E}, \mathscr{G})$ which contain $\Lambda$ and also in all hereditary orders in $\mathscr{A}$ which contain 4 . In particular, one is interested in knowing sufficient conditions for $\Lambda$ itself to be hereditary. This last question is answered by Theorem 1 , and the other, more general question, is succesively reduced to the classical complete case (i.e., when $\mathscr{R}$ is a local complete Dedekind domain and $\mathscr{E}$ is a Galois field extension of $\mathscr{F}$ with group $\mathscr{G}$ ), to the totally ramified case (i.e., when, furthermore, $\mathscr{E} / \mathscr{F}$ is totally ramified) and, finally, to the wildly ramified case.

1. Introduction. In general, we use in this paper the terminology of [3]. With $\mathscr{R}$ we denote a Dedekind domain whose field of quotients is $\mathscr{F}$. $\mathscr{C}$ will be a separable $\mathscr{F}$-algebra and $\mathscr{E}$ a finite dimensional, semisimple, commutative subalgebra of $\mathscr{H}$. We denote with $\mathscr{G}$ a finite subgroup of the group of automorphisms of $\mathscr{E}$.

Let $\mathscr{B}$ be a commutative ring with identity and $\mathscr{G}$ a finite group of authomorphisms of $\mathscr{B}$. Then the group of units of $\mathscr{B}, U(\mathscr{B})$, is a $G$-module. Let $f$ be a factor system: $[f] \in H^{2}(\mathscr{G}, U(\mathscr{B}))$. Then we define the ring $\Delta(\mathfrak{f}, \mathscr{B}, \mathscr{C})$ as a free $\mathscr{B}$-module with basis $\left\{\mathrm{t}_{o}\right\}(\sigma \in \mathscr{G})$, for which a multiplication rule is given by means of:

$$
\left(\mathfrak{b t}_{\sigma}\right)\left(\mathfrak{c t}_{\tau}\right)=\mathfrak{b c} \mathfrak{c}^{\sigma}(\sigma, \tau) \mathfrak{t}_{\sigma \tau} \quad(\mathfrak{b}, \mathfrak{c} \in \mathscr{\mathscr { B }} ; \sigma, \tau \in \mathscr{G})
$$

and extended by additivity.

Definition 1. Given $\mathscr{E}$, a finite dimensional, commutative, semisimple $\mathscr{F}$-algebra, and a finite subgroup $\mathscr{G}$ of the automorphisms of $\mathscr{E}$ such that the fixed subalgebra of $\mathscr{E}$ under the action of $\mathscr{G}$ is $\mathscr{F}: \mathscr{E}^{\mathscr{C}}=\mathscr{F}$, we say that $\mathscr{A}$ is a crossed product of $\mathscr{G}$ over $\mathscr{E}$ when $\mathscr{A}$ is isomorphic to the $\mathscr{F}$-algebra $\Delta(\mathfrak{f}, \mathscr{E}, \mathscr{G})$, for some factor system $f$ corresponding to an element of $H^{2}(\mathscr{G}, U(\mathscr{E}))$.

Definition 2. We say that the $\mathscr{R}$-order $\Lambda$ is a crossed product of $\mathscr{G}$ over $\Lambda_{0}$ when: $\Lambda_{0}$ is an $\mathscr{R}$-order in $\mathscr{E}$, when the fixed subalgebra 
of $\mathscr{E}$ under the action of $\mathscr{G}$ is $\mathscr{F}$, when $\Lambda_{0}$ is stable under $\mathscr{G}$, and when $A$ is isomorphic to $\Delta\left(\mathfrak{f}, \Lambda_{0}, \mathscr{G}\right)$, for some factor system $\mathfrak{f}$ corresponding to an element of $H^{2}\left(\mathscr{G}, U\left(\Lambda_{0}\right)\right)$.

In the case of Definition 1 we usually allow ourselves to write $\mathscr{A}=\Delta(\mathfrak{f}, \mathscr{E}, \mathscr{G})$, and $\Lambda=\Delta\left(\mathfrak{f}, \Lambda_{0}, \mathscr{G}\right)$ in the case of Definition 2. In both cases it is possible to assume, without loss of generality, that $f$ is normalized, which we will do unless it is otherwise stated. If $\mathscr{A}=\Delta(\mathrm{f}, \mathscr{E}, \mathscr{G})$, one can introduce crossed product orders in $\mathscr{A}$ by merely taking an $\mathscr{R}$-order $A_{0}$ in $\mathscr{E}$ which is $\mathscr{G}$-stable and such that $\mathrm{f}(\mathscr{G}, \mathscr{G}) \subset U\left(\Lambda_{0}\right)$, and forming $\Delta\left(\mathfrak{f}, \Lambda_{0}, \mathscr{G}\right)$ within $\mathscr{A}$. What we mean by this is the following: If $\mathscr{A}=\Delta(\mathfrak{f}, \mathscr{E}, \mathscr{C})$, there is a basis $\left\{\mathrm{t}_{\sigma}\right\}$ $(\sigma \in \mathscr{G})$ of the $\mathscr{E}$-module $\mathscr{A}$ such that $\left(\mathrm{xt}_{\sigma}\right)\left(\mathfrak{y t} \mathrm{t}_{\tau}\right)=\mathfrak{r} \mathfrak{y}^{\sigma} \mathrm{f}(\sigma, \tau) \mathrm{t}_{\sigma \tau}$ and, if 1 is the identity of $\mathscr{G}, t_{1}=1$, the identity of $\mathscr{A}$. Such a basis will be called a natural $\mathscr{E}$-basis for $\mathscr{A}$. In a similar way, we have a natural $\Lambda_{0}$-basis for a crossed product order $\Lambda$. In these cases each $\sigma \in \mathscr{G}$ coincides with the restriction to $\mathscr{E}$ (resp. $\Lambda_{0}$ ) of the conjugation by $\mathrm{t}_{\sigma}$. Now, if $\mathscr{A}$ is such a crossed product with such a natural $\mathscr{E}$-basis, given an $\mathscr{R}$-order $\Lambda_{0}$ in $\mathscr{E}$, which is $\mathscr{G}$-stable and such that $\mathrm{f}(\mathscr{G}, \mathscr{G}) \subset U\left(\Lambda_{0}\right)$ it is enough to form $A=\sum_{\sigma \in \mathscr{G}} \Lambda_{0} \mathrm{t}_{\sigma}=\bigoplus_{\sigma \in \mathscr{G}} \Lambda_{0} \mathrm{t}_{\sigma}$ to have a crossed product order in $\mathscr{A}$ which is isomorphic to $\Delta\left(\mathfrak{f}, \Lambda_{0}, \mathscr{G}\right)$.

Also, given a crossed product order $\Lambda=\Delta\left(\mathfrak{f}, \Lambda_{0}, \mathscr{G}\right)$, it is an order in a crossed product algebra of the form $\Delta(\mathfrak{f}, \mathscr{C}, \mathscr{G})$, which is obtained as the usual algebraic container: $\mathscr{A}=\mathscr{F} \otimes_{\mathscr{R}} \Lambda$.

It is an easy exercise to prove that a crossed product algebra is centrally simple, and hence separable (cf. [2]). If $\mathscr{A}$ is a crossed product, let $\mathscr{E}_{i}(i=1, \cdots, m)$ be the simple components of $\mathscr{E}$, and $1=\mathfrak{e}_{1}+\cdots+\mathfrak{e}_{m}$ the corresponding decomposition into primitive orthogonal idempotents. The requirement that $\mathscr{E}^{\mathscr{S}}=\mathscr{F}$ in Definition 1 implies that $\mathscr{G}$ operates transitively on $\left\{e_{1}, \cdots, e_{m}\right\}$. Each $\mathscr{E}_{\mathfrak{i}}$ is a field, which is Galois over $\mathscr{F}_{\mathfrak{e}_{i}}$ with Galois group $\mathscr{H}_{i}=\operatorname{Stab}_{\mathscr{E}}\left(\mathfrak{e}_{i}\right)$, and each pair $\mathscr{E}_{\mathrm{i}}, \mathscr{E}_{\mathrm{i}}$ is a pair of fields conjugate with respect to $\mathscr{F}$. Sometimes, by abuse of language, we say that $\mathscr{E}_{i}$ is Galois over $\mathscr{F}$ with group $\mathscr{K}_{\mathfrak{i}}$. If $\mathfrak{n}$ denotes the order of $\mathscr{G}$, the dimension of $\mathscr{E}$ over $\mathscr{F}$ and the rank of $\mathscr{A}$ over $\mathscr{E}$ are both equal to $\mathfrak{n}$, while the dimension of $\mathscr{A}$ over $\mathscr{T}$ is $\mathfrak{n}^{2}$. It may be noted (which is also easily proven) that $\mathscr{E}$ is a maximal commutative subalgebra of $\mathscr{A}$.

We will refer to the case in which $\mathscr{E}$ is simple (i.e., when it is a field) by saying that we have a classical crossed product algebra or a classical crossed product order.

2. Hereditary crossed product orders. 
TheOREM 1. Let $\mathscr{R}$ be a local complete Dedekind domain with field of quotients $\mathscr{F}$. If the extension $\mathscr{E}_{1} / \mathscr{F}$ is tamely ramified and if $\Lambda_{0}$ is the maximal $\mathscr{R}$-order $\Omega$ in $\mathscr{E}$, then $\Lambda=\Delta\left(\mathfrak{f}, \Lambda_{0}, \mathscr{G}\right)$ is $a$ hereditary order and, moreover, a principal hereditary order (for the definition of it, see [6]).

This result, which is essentially equivalent to a theorem of S. Williamson (cf. [5]), is, in a more general form, a corollary of Proposition 1, except for the part that affirms that the hereditary order is principal. We give a direct and easy proof, based on S. Williamson's result.

Proof. By calling $f_{1}$ the function $e_{1} \cdot f$ and $\mathscr{A}_{1}$ the algebra $e_{1} \mathscr{A} e_{1}$, we have that $\mathscr{A}_{1}$ is a crossed product $\Delta\left(\mathfrak{f}_{1}, \mathscr{E}_{1}, \mathscr{C}_{1}\right)$ that is a classical crossed product. If we call $\Lambda_{1}$ the $\mathscr{R}$-order $\mathrm{e}_{1} \Lambda \mathrm{e}_{1}$, we see that it is a classical crossed product order $\Delta\left(\mathfrak{f}_{1}, \Lambda_{01}, \mathscr{C}_{1}\right)$ in $\mathscr{A}_{1}$, where $\Lambda_{01}$ is the $\mathscr{R}$-order $\mathrm{e}_{1} \Lambda_{0}$ in the field $\mathscr{E}_{1}$.

According to S. Williamson's result, $\Lambda_{1}$ is principal hereditary if $\mathscr{E}_{1} / \mathscr{F}$ is tamely ramified. In this case, the radical of $\Lambda_{1}, \mathscr{N}_{1}$, is of the form: $\mathscr{N}_{1}=\pi_{1} \Lambda_{1}$, where $\pi_{1}$ is a prime element of $\Lambda_{01}$ (and, hence, $\pi_{1}$ generates the radical of $\Lambda_{01}$, which is its unique maximal ideal). Let $\sigma_{1}=1, \sigma_{2}, \cdots, \sigma_{m}$ be a system of representatives of the cosets $\sigma_{i} \mathscr{H}$ of $\mathscr{G}$-modulo $\mathscr{H}=\mathscr{H}_{1}$ such that $\sigma_{1}\left(\mathrm{e}_{1}\right)=\mathrm{e}_{\mathrm{i}}$, and then define $\pi$ to be the element of $\Lambda_{0}$ obtained by:

$$
\pi=\sum_{i=1}^{m} \sigma_{i}\left(\pi_{1}\right) \text {. }
$$

Let $\mathscr{N}$ be the radical of $\Lambda$. Theorem 1 will be proved if we succeed in showing that $\mathscr{N}=\pi \Lambda=\Lambda \pi$. The second equality is clear, in view of the definition of $\pi$. It follows readily from Nakayama's lemma that $\pi \Lambda \subset \mathscr{N}$. To prove the reverse inclusion, we form the quotient

$$
\frac{\Lambda}{\pi \Lambda}=\bar{\Lambda}
$$

which is in an obvious way of the form

$$
\Delta\left(\overline{\mathrm{f}}, \frac{\Lambda_{0}}{\pi \Lambda_{0}}, \mathscr{G}\right)
$$

(bars denoting objects modulo $\pi$ ), and show that $\operatorname{rad}(\bar{\Lambda})=0$.

But here we notice at once that $(\bar{\Lambda})_{1}=\left(\overline{\Lambda_{1}}\right)$, where the left-hand side means $\bar{e}_{1} \bar{\Lambda}_{1} \bar{e}_{1}$ and the right-hand side means $\Lambda_{1} /\left(\pi_{1} \Lambda_{1}\right)=\Lambda_{1} / \mathscr{N}_{1}$. 
Hence, $\bar{\Lambda}_{1}$ is semisimple. Now, if we express elements of $\bar{\Lambda}_{0}$ as the corresponding sums of their projections into the simple components, an arbitrary element $\bar{\delta}$ in $\operatorname{rad}(\bar{\Lambda})$ is of the form

$$
\bar{\delta}=\sum_{i, \sigma} \alpha_{i, \sigma} \bar{e}_{i} \bar{t}_{\sigma}
$$

and a direct calculation shows that (for each $\mathfrak{i}, j) \overline{\mathfrak{t}}_{o_{i}}^{-1} \bar{e}_{i} \bar{\delta}_{\mathrm{e}_{1}} \overline{\mathfrak{t}}_{o_{1}}$ is equal to 0 because it is an element of $\operatorname{rad}(\bar{\Lambda}) \cap \bar{\Lambda}_{1} \subset \operatorname{rad}\left(\bar{\Lambda}_{1}\right)$. It follows that all the $\alpha_{\mathrm{i}, \sigma}$ are 0 , so that $\bar{\delta}=0$.

2. Reduction to the local case. In this section we assume $\mathscr{R}$ is a local Dedekind domain and we keep the preceeding notations and conventions. We assume further that $\Lambda_{0}$ is equal to the sum of its simple components (meaning that all the idempotents $e_{i}$ are elements of $\Lambda_{0}$ ).

Let us be given a crossed product order $\Lambda=\Delta\left(\mathfrak{f}, \Lambda_{0}, \mathscr{G}\right)$ in the crossed product algebra $\mathscr{A}=\Delta(\mathfrak{f}, \mathscr{E}, \mathscr{G})$. We considerar a $\Lambda$-ideal, $\mathscr{L}$, i.e., a full $\mathscr{R}$-lattice in $\mathscr{A}$ such that $\Lambda \mathscr{M} \subset \mathscr{M}$ and $\mathscr{M} \Lambda \subset \mathscr{M}$. In particular, $\mathscr{C}$ may be any order containing $\Lambda$. We define, $\mathscr{C}_{1}=$ $\mathrm{e}_{1} \mathscr{M} \mathrm{e}_{1}$. It is immediate then that $\mathscr{L}_{1}=\mathscr{M} \cap \mathscr{A}_{1}$.

LEMma 1. We have:

$$
\mathscr{C l}=\Lambda \mathscr{C}_{1} \Lambda=\sum_{\sigma, \tau} \mathrm{t}_{\sigma} \mathscr{C}_{1} \mathbf{t}_{\tau} .
$$

Proof. It is clear that $\mathscr{M}_{1}$ is a $\Lambda_{0}$-module (left and right). Next, given an element $x$ of $\mathscr{A}$, we have through a direct computation that

$$
\mathfrak{t}_{\sigma_{\mathrm{i}}-1} \mathfrak{e}_{\mathrm{i}} \boldsymbol{x e}_{\mathrm{i}} \mathrm{t}_{\sigma_{\mathrm{i}}} \in \mathscr{M}_{1}
$$

so that elements:

$$
\mathfrak{x}_{\mathrm{ii}}=\frac{1}{\mathrm{f}\left(\sigma_{\mathrm{i}}, \sigma_{\mathrm{i}}^{-1}\right)^{\sigma_{\mathrm{i}}^{-1}}} \cdot t_{\sigma_{\mathrm{i}}^{-1}} \mathrm{e}_{\mathrm{i}} \mathrm{x}_{\mathrm{i}} \mathrm{t}_{\sigma_{\mathrm{i}}} \cdot \frac{1}{\mathrm{f}\left(\sigma_{\mathrm{i}}, \sigma_{\mathrm{i}}^{-1}\right)^{\sigma_{\mathrm{i}}^{-1}}}
$$

are in $\mathscr{C}_{1}$ and are such that

$$
\mathfrak{x}=\sum_{i, j} \mathrm{t}_{\sigma_{i}} \mathfrak{x}_{\mathrm{ii}} \mathrm{t}_{\sigma_{i}}^{-1}
$$

This shows that $\mathscr{C} \subset \Lambda \mathscr{C}_{1} \Lambda \subset \mathscr{C}$, which gives the desired result.

Note. For this result one does not need to assume that $\mathscr{R}$ is local.

Proposition 1. Let $\mathscr{J}$ be the set of all 1 -ideals in $\mathscr{A}$ and $\mathscr{F}_{1}$ 
the set of-all $\Lambda_{1}$-ideals in $\mathscr{A}_{1}$. We introduce the following mappings:

$$
\begin{aligned}
& R: \mathscr{I} \longrightarrow \mathscr{I}_{1} ; R(\mathscr{A})=\mathscr{M}_{1} \cdot \\
& S: \mathscr{I}_{1} \longrightarrow \mathscr{I} ; S\left(\mathscr{M}_{1}\right)=\Lambda \mathscr{N}_{1} \Lambda .
\end{aligned}
$$

Then $R$ and $S$ are inverse bijections and have the following properties:

(i ) $\mathscr{H} \subset \mathscr{M}^{\prime} \Rightarrow R(\mathscr{H}) \subset R\left(\mathscr{L}^{\prime}\right)$.

(ii) $\Gamma \in \mathscr{J}$ is an order if and only if $R(\Gamma)$ is an order.

(iii) If $\Gamma \in \mathscr{J}$ is an order, then $\mathscr{L}$ is a $\Gamma$-ideal if and only if $R(\mathscr{L})$ is an $R(\Gamma)$-ideal. In particular, $\operatorname{rad}(R(\Gamma))=R(\operatorname{rad}(\Gamma))$, $\operatorname{rad}(S(\Gamma))=S(\operatorname{rad}(\Gamma))$.

(iv) $\Gamma \in \mathscr{I}$ is a maximal (resp. hereditary) order if and only if $R(\Gamma)$ is a maximal (resp. hereditary) order.

Proof. (i) clear. (ii) If $\Gamma$ is an order containing $\Lambda$, it is clear that $\Gamma_{1}$ is a finitely generated $\mathscr{R}$-module which is a ring containing $\Lambda_{1}$ and, hence generates $\mathscr{A}_{1}$ under $\mathscr{F}$. Conversely, if $\Gamma_{1}$ is an order in $\mathscr{A}_{1}$ containing $\Lambda_{1}, S\left(\Gamma_{1}\right) \supset S\left(\Lambda_{1}\right)=\Lambda$, whence $S\left(\Gamma_{1}\right)$ is a finitely generated $\mathscr{R}$-module containing $\Lambda$. Its closure under multiplication follows from: $\Lambda \Gamma_{1} \Lambda \Lambda \Gamma_{1} \Lambda=\Lambda \Gamma_{1} \mathrm{e}_{1} \Lambda \mathrm{e}_{1} \Gamma_{1} \Lambda=\Lambda \Gamma_{1} \Lambda_{1} \Gamma_{1} \Lambda=\Lambda \Gamma_{1} \Lambda$. (iii) If $\mathscr{L}$ is a $\Gamma$-ideal, $\Gamma \mathscr{L}=\mathscr{L} \Gamma=\mathscr{L}$ implies: $\quad \Gamma_{1} \mathscr{L}_{1}=\mathrm{e}_{1} \Gamma \mathrm{e}_{1} \mathrm{e}_{1} \mathscr{L} \mathrm{e}_{1} \subset$ $\mathrm{e}_{1} \Gamma \mathscr{L} \mathrm{e}_{1}=\mathscr{L}_{1}$ and, similarly, $\mathscr{L}_{1} \Gamma_{1} \subset \mathscr{L}_{1}$. The converse is equally easy. (iv) Let $\Gamma$ be a hereditary order containing $\Lambda$ and let us call $\mathscr{N}$ the radical of $\Gamma$. We also introduce:

$$
\left.\mathscr{N}^{-1}=\{\mathfrak{x} \in \mathscr{A} / \mathfrak{x} \mathscr{N} \subset \Lambda\}, \mathscr{N}_{1}=\operatorname{rad}\left(\Gamma_{1}\right)\right)
$$

and $\mathscr{N}_{1}^{-1}=\left\{x \in \mathscr{A} / \mathfrak{x} \mathscr{N}_{1}^{-1} \subset \Gamma_{1}\right\}$. Then, there exist two finite families: $\mathfrak{x}_{i}, \mathfrak{y}_{i}$, such that $\mathfrak{x}_{i} \in \mathscr{N}, \mathfrak{y}_{i} \in \mathscr{N}^{-1}$ and $\sum \mathfrak{y}_{i} \mathfrak{x}_{i}=1$. It follows that the elements $\mathrm{t}_{\sigma_{q}}^{-1} e_{q} x_{i} e_{1}$ are in $\mathscr{N}_{1}$, and the elements $\mathrm{e}_{1} \mathfrak{y}_{\mathrm{i}} \mathrm{e}_{q} \mathrm{t}_{\sigma_{q}}$ in $\mathscr{N}_{1}^{-1}$. It follows easily that $\mathscr{N}_{1}^{-1} \mathscr{N}_{1}=\Gamma_{1}$. This suffices to say that $\mathscr{N}_{1}$ is a projective $\Gamma_{1}$-module, which is a necessary and sufficient condition for an order (in the local case) to be hereditary. Conversely, if $\Gamma_{1}$ is hereditary (that is, if $\mathscr{N}_{1}^{-1} \mathscr{N}_{1}=\Gamma_{1}$ ) it follows: $\Lambda \mathscr{N}_{1}^{-1} \Lambda \cdot \Lambda \mathscr{N}_{1} \Lambda=\Lambda \mathscr{N}_{1}^{-1} \Lambda_{1} \mathscr{N}_{1} \Lambda=$ $\Lambda \mathscr{N}_{1}^{-1} \mathscr{N}_{1} \Lambda=\Lambda \Gamma_{1} \Lambda=\Gamma$, which is enough to guarantee that $\Gamma$ is hereditary.

Note. The assumption that $\mathscr{R}$ is local is used only in the proof of part (iv) in the preceeding theorem.

CoRollary. . Let $\Lambda_{0}=\Omega$, the unique maximal order in the commutative algebra $\mathscr{E}$. Then the following propositions are equivalent:

(i) $\Lambda$ is hereditary for every factor system f;

(ii) if $\mathrm{f}=1, \Lambda$ is hereditary; 
(iii) $\mathscr{E}_{1}$ is a tamely ramified extension of $\mathscr{F}$. If, furthermore, the residue class field of $\Lambda_{01}$ is perfect, then the propositions above are equivalent to the following:

(ii)' There exists a factor system f such that $\Lambda$ is hereditary.

Proof. (i) implies (ii), and (ii) implies that $\Delta(1, \mathscr{E}, \mathscr{G})_{1}$ is hereditary which, by S. Williamson's results (cf. [5]) implies (iii). Again, by S. Williamson's results, (iii) implies that $\Lambda_{1}$ is hereditary, which implies (i). (ii)' implies that $\Delta(\mathfrak{f}, \mathscr{E}, \mathscr{G})_{1}$ is hereditary for some $f$. Then, if $\Lambda_{01}$ has a perfect residue class field, by Harada's results (cf. [1]), (iii) follows from (ii)'. Clearly, (i) implies (ii)'.

Remark on the possibility of an effective computation of the Schur index. Let $\mathscr{R}$ be a local complete Dedekind domain and let us assume that $\mathscr{E}_{1} / \mathscr{F}$ is tamely ramified. We are going to show how, using only the information contained in the data for the definition of $\Lambda$ as a crossed product order, it is possible to compute the Schur index of both $\mathscr{A}$ and $\mathscr{A}_{1}$ (which, as it turns out, are equal to each other).

Let us work first under the simplifying assumption that we are in the classical case: $\mathscr{A}=\mathscr{A}_{1}, \mathscr{E}=\mathscr{E}_{1}$. The centrally simple algebra $\mathscr{A}$ can be viewed as a matrix algebra of degree $\mathfrak{k}$, say, over a division algebra $\mathscr{D}$. Since $\Lambda$ is a principal hereditary order, it is a block order corresponding to a matrix of the form:

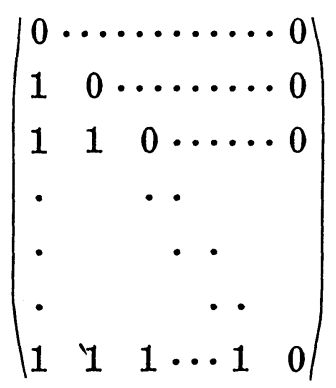

whose blocks are all of the same size ( $x \times \mathfrak{x}$, say) (cf. [3], Ch. IX, §2). In [5], S. Williamson has proved that if $t$ is the number of blocks (and, hence $\mathfrak{t}=\mathfrak{x t}$ ) then $t$ can be computed as the order of the conductor group of [f], $H_{[\mathrm{f}]}$ which depends only on [f].

It is well known that $\mathscr{D}$ has a unique maximal order $\hat{\Omega}$ with a unique maximal ideal $\hat{\mathscr{N}}$ and that the Schur index of $\mathscr{A}$ (which is, by definition, the square root of the $\mathscr{F}$-dimension of $\mathscr{D}), \mathfrak{B}$, is equal to the dimension of $\overline{\mathscr{D}}=\hat{\Omega} / \hat{\mathscr{N}}$ over $\overline{\mathscr{F}}=\mathscr{R} / \operatorname{rad}(\mathscr{R})$. Let us introduce the notation $\mathfrak{f}_{\pi}$ for the residue class degree of $\mathscr{E}$ over $\mathscr{F}$. Then we can look at the quotient $\bar{\Lambda}=\Lambda / \mathscr{N}$ in two different ways, 
namely as a direct sum of $\mathfrak{n}=(\mathscr{G}: 1)$ copies of $\overline{\mathscr{E}}=\Omega / \operatorname{rad}(\Omega)$ and as the direct sum of "residue matrix blocks" which arises when we compute the quotient starting from the general form of the matrices in 4 . A computation of the $\overline{\mathscr{F}}$-dimension of $\bar{\Lambda}$ from each point of view gives:

$$
\operatorname{trg}^{2} \mathfrak{S}=\mathfrak{n} \mathfrak{f}_{\pi}
$$

from which we get that:

$$
\mathfrak{x}=\mathrm{f}_{\pi} .
$$

Then, we obtain the desired result in the form of the formula:

$$
\mathfrak{s}=\frac{\mathfrak{n}}{\mathfrak{f}_{\pi} \mathfrak{t}} .
$$

If we proceed, for the general case, in a similar way, we get that the Schur index of $\mathscr{A}$ is equal to the Schur index of $\mathscr{A}_{1}$.

Remark on the reduction to the complete case. Assume once again that $\mathscr{R}$ is a local Dedekind domain. Questions regarding the hereditarity or maximality of orders in $\mathscr{A}$ containing $\Lambda$ can be reduced to the same questions concerning orders in $\hat{A}$ containing the completion $\hat{\Lambda}$ of $\Lambda$. However, the completion $\hat{A}$ of a classical - crossed product $\mathscr{A}=\Delta(\mathfrak{f}, \mathscr{E}, \mathscr{G})$ is in general no longer a classical crossed product, but rather a crossed product in the sense of our Definition 1 (see Proposition 1.4 of [5]). More precisely, $\hat{A}=$ $\Delta(\mathfrak{f}, \hat{\mathscr{E}}, \mathscr{G})$ where $\hat{\mathscr{E}}=\mathscr{E}_{1} \oplus \cdots \oplus \mathscr{E}_{3}$ and the $\mathscr{E}_{\mathfrak{i}}$ denote the completions of $\mathscr{E}$ at each of the prime ideals $\mathscr{P}_{i}$ lying over the prime ideal of $\mathscr{F}$. The rest of the paper is devoted to reducing the study of $\Lambda$ in $\mathscr{A}$ to the local complete case, namely to the study of $\hat{\Lambda_{1}}$ in $\hat{\mathscr{A}}_{1}=\Delta\left(\mathfrak{f}_{1}, \mathscr{E}_{1}, \mathscr{Z}_{1}\right)$ where $\mathscr{Z}_{1}$ denotes the decomposition group of $\mathscr{P}_{1}$.

3. Reduction to the totally ramified case. Throughout this section we keep our general notations but we assume that $\mathscr{R}$ is a local complete Dedekind ring and that $\mathscr{E} / \mathscr{F}$ is a Galois extension with Galois group $\mathscr{G}$.

We fix a normal subgroup $\mathscr{H}$ of $\mathscr{G}$ and call $\mathscr{L}$ the fixed field of $\mathscr{H}$ so that $\mathscr{E} / \mathscr{L}$ is Galois with group $\mathscr{H}$ and $\mathscr{L} / \mathscr{F}$ is Galois with group $\mathscr{G} / \mathscr{H}$.

We use the following natations:

$\pi$ is a prime element of $\mathscr{R}$, so that $\operatorname{rad}(\mathscr{R})=\pi \mathscr{R}$.

$\overline{\mathscr{F}}=\mathscr{R} / \pi \mathscr{R}$.

$\Lambda_{0 \mathscr{C}}=\Lambda_{0} \cap \mathscr{L}=\Omega=$ the maximal $\mathscr{R}$-order in $\mathscr{L}$.

$\Lambda_{0}^{\mathscr{P}}=\Omega \otimes_{\mathscr{R}} \Lambda_{0}$. 


$$
\begin{aligned}
& \mathscr{E}^{\mathscr{H}}=\mathscr{L} \boldsymbol{\otimes}_{\mathscr{F}} \mathscr{E} . \\
& \mathscr{A}^{\mathscr{C}}=\mathscr{L} \boldsymbol{Q}_{\mathscr{T}} \mathscr{A} . \\
& \Lambda^{\mathscr{H}}=\Omega \boldsymbol{\otimes}_{\mathscr{T}} \Lambda . \\
& \sigma_{1}=1, \sigma_{2}, \cdots, \sigma_{\mathfrak{m}} \text { are representatives of the cosets of } \mathscr{G} \text {-modulo } \\
& \mathscr{H}, \text { or of the elements of } \mathscr{C} / \mathscr{H} .
\end{aligned}
$$

We assume that $\mathscr{A}$ is identified to the subalgebra $1 \otimes \mathscr{A}$ of $\mathscr{A}^{\text {Se }}$.

LEMMA 2. If $\mathscr{L} / \mathscr{F}$ is an unramified extension, then $\Lambda_{0}^{\mathscr{C}}$ is equal to the sum of its simple components.

Proof. The $\mathscr{F}$-algebra $\mathscr{L} \bigotimes_{\sigma} \mathscr{E}$ is semisimple and it contains in an obvious way the semisimple $\mathscr{F}$-algebra $\mathscr{L} \otimes_{\mathscr{F}} \mathscr{L}$ and both have the same primitive orthogonal idempotents. Let $\left(\mathfrak{y}_{\mathfrak{i}}\right)(\mathfrak{i}=1, \cdots, \mathfrak{m})$ be an $\mathscr{R}$-basis of $\Omega$ and let $\Delta$ be the discriminant of this basis. The standard decomposition of a tensor product of $\mathscr{F}$-extensions: $\mathscr{K} \otimes_{\mathscr{F}} \mathscr{L}$, where $\mathscr{L} / \mathscr{F}$ is separable, through the chinese remainder theorem allows for a direct computation of the irreducible idempotents $e_{i}$. In our case, one obtains:

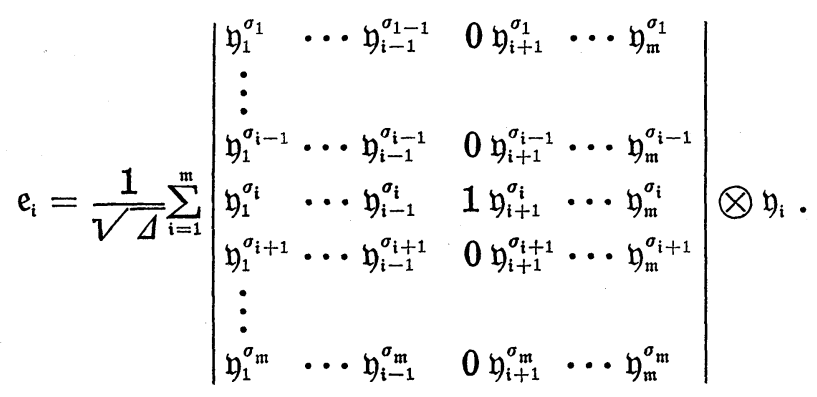

Now, if $\mathscr{L} / \mathscr{F}$ is unramified, $\Delta$ is a unit and the lemma follows.

The group $\mathscr{G}$ may be considered as a group of automorphisms of the semisimple commutative $\mathscr{F}$-algebra $\mathscr{E}^{\mathscr{C}}$, provided that we define the action of $\sigma \in \mathscr{G}$ in $\mathscr{E}^{\mathscr{C}}$ by establishing that $(\mathfrak{x} \otimes \mathfrak{y})^{\sigma}=$ $\mathfrak{x} \otimes \mathfrak{y}^{\sigma}(\mathfrak{x} \in \mathscr{L}, \mathfrak{y} \in \mathscr{E}, \sigma \in \mathscr{G})$. On the other hand, the factor system $\mathfrak{f}$ may be viewed as a factor system $\mathrm{f}: \mathscr{G} \times \mathscr{G} \rightarrow \mathscr{E}^{\mathscr{P}}$ (by identifying each $f(\sigma, \tau)$ with $1 \otimes f(\sigma, \tau)$ ). If we do this, we can state:

Lemma 3. $\mathscr{A}^{\mathscr{K}}$ is a crossed product $\Delta(\mathfrak{f}, \mathscr{E} \mathscr{\mathscr { C }}, \mathscr{G})$.

Proof. Straightforward.

We introduce the following sets:

$\mathscr{O}^{\mathscr{C}}$ is the set of all orders $\Gamma$ in $\mathscr{A}^{\mathscr{C}}$ which contain $\Lambda^{\mathscr{K}}$ and verify 
the following condition:

for each element $\mathfrak{x}$ in $\Gamma$ there is a representation of the form

$$
\mathfrak{x}=\sum_{i} \mathfrak{w}_{\mathfrak{i}} \otimes \mathfrak{x}_{\mathfrak{i}}
$$

(with $\mathfrak{w}_{\mathfrak{i}} \in \Omega, \mathfrak{x}_{\mathfrak{i}} \in \mathscr{A}$ ) such that each $1 \otimes \mathfrak{x}_{i}$ is also an element of $\Gamma$.

$\mathcal{O}$ is the set of all orders in $\mathscr{A}$ which contain $A$.

Next, we consider the following two functions:

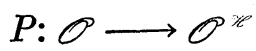

$$
\begin{aligned}
& P(\Gamma)=\Omega \otimes_{\mathscr{R}} \Gamma=: \Gamma^{x} \\
& I: O^{\mathscr{C}} \longrightarrow O \\
& I(\Gamma)=\Gamma \cap \mathscr{A} \text {. }
\end{aligned}
$$

Then we have:

PRoposition 2. $P$ and I are order preserving bijections, inverses of each other.

If, furthermore, $\mathscr{L} \mid \mathscr{F}$ is an unramified extension, the following diagram is commutative:

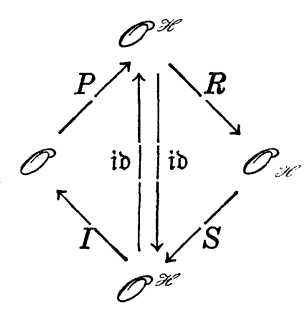

where $R$ and $S$ are the mappings which were introduced in the Proposition 1, applied to the case in which $\mathscr{A}$ is $\mathscr{A}^{\mathscr{a}}$ and, thus, $\mathscr{A}_{1}$ is $\left(\mathscr{C}^{\mathscr{C}}\right)_{1}=: \mathscr{A}_{\mathscr{C}}=: \Delta(\mathrm{f} \mid \mathscr{H} \times \mathscr{H}, \mathscr{E}, \mathscr{H})$. If follows that $\mathscr{O}_{\mathscr{H}}$ (the image of $\mathscr{O}^{\mathscr{C}}$ under $R$ ) is the set of all $\mathscr{R}$-orders in $\mathscr{X}_{\mathscr{H}}$ which contain

$$
\Lambda_{\mathscr{C}}=\Delta\left(\mathrm{f} \mid \mathscr{H} \times \mathscr{H}, \Lambda_{0}, \mathscr{H}\right) .
$$

Furthermore, in this latter situation both $P$ and I carry hereditary orders into hereditary orders.

Proof. Most of the proof is direct. For example, to prove that $P \circ I=$ ib, we take $\Gamma \in \mathcal{O}^{\mathscr{C}}$ and form $\Omega \otimes_{\mathscr{T}}(\Gamma \cap \mathscr{A})$, which has to be shown is equal to $\Gamma$. It is obviously contained in $\Gamma$. But also, if $\gamma \in \Gamma$, according to $\left(^{*}\right)$, we can write it in the form: $\sum_{i} \mathfrak{w}_{i} \otimes \mathfrak{x}_{i}$, 
with each $1 \otimes r_{i}$ belonging to $\Gamma$. But this actually means that each $1 \otimes \mathfrak{x}_{i}$ belongs to $\Gamma \cap \mathscr{A}$, whence $\gamma \in \Omega \otimes_{\mathscr{R}}(\Gamma \cap \mathscr{A})$. The fact that the other composite, $I \circ P$, is the identity of $\odot$ goes as follows: Let $\Gamma \in \mathcal{O}$; then $\Gamma \subset\left(A_{0 \mathscr{C}} \otimes \Gamma\right) \cap \mathscr{A}$. Now, if $\gamma$ is in the latter order and if $\mathfrak{w}_{i}$ is an $\mathscr{R}$-basis of $\Omega$, then there are elements $\mathfrak{r}_{i}$ in $\Gamma$ such that $\gamma=\sum_{\mathrm{i}} \mathfrak{w}_{\mathrm{i}} \otimes \mathfrak{r}_{\mathrm{i}}=1 \otimes \mathfrak{x}$ (with $\mathfrak{x}$ in $\left.\mathscr{A}\right)$. Then, if $\alpha_{\mathrm{i}}$ are elements of $\mathscr{L}$ such that $1=\sum_{\mathrm{i}} \alpha_{\mathrm{i}} \mathfrak{w}_{\mathrm{i}}$, it follows that $\alpha_{\mathrm{i}} \mathrm{x}_{\mathrm{i}}=\mathfrak{x}_{\mathrm{i}} \in \Gamma$, which implies that $\mathfrak{x}=\sum_{\mathfrak{i}} \mathfrak{w}_{\mathfrak{i}} \mathfrak{x}_{\mathfrak{i}} \in \Gamma$.

In the case that $\mathscr{L} / \mathscr{F}$ is unramified, according to Lemma 1 , $\Lambda_{0}^{*}$ is the sum of its simple components and we can apply Proposition 1 to our situation in which $\mathscr{A}^{\mathscr{C}}$ plays the role of $\mathscr{A}$, and $\Lambda^{\mathscr{C}}$ the role of $\Lambda$.

As for the statement on the behavior of $P$ and $I$ in this unramified case, we proceed as follows. Let $\mathscr{N}, \mathscr{N}^{\mathscr{C}}$, be the radicals of $\Gamma$ and $P(\Gamma)=\Gamma^{\mathscr{C}}$ and let

$$
\mathscr{N}^{-1}=\{x \in \mathscr{L} / \mathfrak{x} \mathscr{N} \subset \Gamma\},\left(\mathscr{N}^{\mathscr{H}}\right)^{-1}=\left(\mathfrak{x} \in \mathscr{A}^{\mathscr{C}} / \mathfrak{x} \mathscr{N}^{\mathscr{H}} \subset \Gamma^{\mathscr{C}}\right\} .
$$

We claim that $\mathscr{N}^{\mathscr{C}}=P(\mathscr{N})=: \Omega \otimes_{\mathscr{R}} \mathscr{N}$. In fact, $\mathscr{N}$ is $\pi$-nilpotent (meaning that for some integer $m, \mathscr{N}^{m} \subset \pi \Gamma$ ), so that $\Omega \otimes_{\mathscr{A}} \mathscr{N}$ is $\pi$-nilpotent too and hence $\Omega \otimes_{\mathscr{R}} \mathscr{N} \subset \mathscr{N}^{\mathscr{C}}$. On the other hand, the assumption that $\mathscr{L} / \mathscr{F}$ is unramified forces $\Omega / \pi \Omega$ to be a separable extension of $\mathscr{F}$ and from this it follows readily that $\Gamma^{\mathscr{P}} /\left(\Omega_{0 \mathscr{C}} \boldsymbol{\otimes}_{\mathscr{A}} \mathscr{N}\right)$ is semisimple, and hence that $\mathscr{N}^{*} \subset \Omega \bigotimes_{\mathscr{R}} \mathscr{N}$.

It is then easy to see that also $\left(\mathscr{N}^{\mathscr{C}}\right)^{-1}=P\left(\mathscr{N}^{-1}\right)=: \Omega \otimes_{\mathscr{\Omega}} \mathscr{N}^{-1}$. Now, if $\Gamma$ is hereditary, we have $\mathscr{N}^{-1} \mathscr{N}=\Gamma$, from which it follows that $\left(\mathscr{N}^{\mathscr{C}}\right)^{-1} \cdot \mathscr{N}^{\mathscr{C}}=\Gamma^{\mathscr{C}}$ which says that $\Gamma^{\mathscr{C}}$ is hereditary. Conversely, if $\Gamma^{\mathscr{C}}$ is hereditary, $\left(\mathscr{N}^{\mathscr{H}}\right)^{-1} . \mathscr{N}^{\mathscr{C}}=\Gamma^{\mathscr{C}}$ and then we have also that $\mathscr{N}^{-1} \mathscr{N}=\Gamma$, or that $\Gamma$ is hereditary. This completes the proof.

Let us remark before proceeding any further that the algebra $\mathscr{A}_{\mathscr{C}}=\Delta(\mathrm{f} \mid \mathscr{H} \times \mathscr{H}, \mathscr{C}, \mathscr{H})$ is a crossed product of the $\mathscr{L}$-algebra $\mathscr{E}$ and either $\Lambda_{\mathscr{C}}$ or any other $\mathscr{R}$-order in $\mathscr{O}_{\mathscr{C}}$ is at the same time an order over the Dedekind ring $\Omega=\Lambda_{0 \mathscr{C}}$. Also, it is important to keep in mind that questions of maximality or hereditarity for elements of $\mathcal{O}_{\mathscr{H}}$ are the same independently that one considers them as $\mathscr{R}$ orders or as $\Omega$-orders.

Let $\mathcal{O}_{\mathscr{C}}^{\prime}$ be the family of all orders in $\mathscr{O}_{\mathscr{C}}$ which are invariant under $\mathscr{G}$, i.e., the set of all orders $\Gamma$ in $\mathscr{O}_{\mathscr{C}}$ for which it is true that:

$$
\mathrm{t}_{\sigma} \Gamma \mathrm{t}_{\sigma}^{-1} \subset \Gamma \quad(\forall \sigma \in \mathscr{C})
$$

and let us introduce the following two functions: 


$$
\begin{aligned}
& J: \mathscr{O} \mathscr{O}_{\mathscr{C}}^{\prime} \\
& J(\Gamma)=\Gamma \cap \mathscr{X}_{\mathscr{C}} \\
& p: \mathscr{O}_{\mathscr{H}}^{\prime} \longrightarrow \mathscr{O} \\
& p(\Gamma)=\Gamma \Lambda=\Lambda \Gamma \Lambda .
\end{aligned}
$$

Then we have:

Proposition 3. $J_{0} p$ is the identity map of $\mathcal{O}_{\mathscr{B}}^{\prime}$ and, for each $\Gamma$ in $\mathcal{O}, p(J(\Gamma))$ is the minimum of the elements of $\mathcal{O}$ whose under $J$ is $J(\Gamma)$.

If $\mathscr{L} / \mathscr{F}$ is unramified, the following diagrams are commutative:
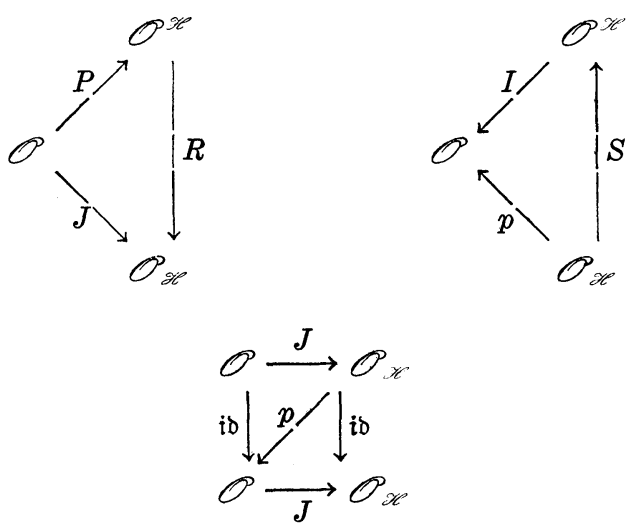

and therefore $\mathcal{O}_{\mathscr{K}}^{\prime}=\mathcal{O}_{\mathscr{H}}$ and both bijections $p$ and $J$ preserve hereditary orders.

Proof. It is clear that $J$ is a mapping from $\mathbb{Q}$ into $\mathcal{O}_{\mathscr{C}}^{\prime}$, because $\mathscr{A}_{\mathscr{C}}$ is invariant under $\mathscr{G}$. It is also easy to see that, for $\Gamma$ in $\mathcal{O}_{\mathscr{C}}^{\prime}, \Gamma \Lambda=\sum_{\sigma} \Gamma \mathrm{t}_{\sigma}$, from which one obtains readily that $p(\Gamma)$ is an element of $\mathcal{O}$. The statements about the composites $J \circ p$ and $p \circ J$ are proved straightforwardly.

Let us assume that $\mathscr{L} / \mathscr{F}$ is unramified and let us take $\Gamma$ in 0 . Then $R(P(\Gamma))$ is the set of all elements of the form

$$
\sum_{i, \sigma} \mathfrak{w}_{i} \mathfrak{x}_{i j} \mathfrak{t}_{\sigma}
$$

such that

$$
\mathfrak{w}_{i} \in \Omega, \quad \sum_{\sigma} \mathfrak{x}_{\mathrm{i} i \sigma} \mathrm{t}_{\sigma} \in \Gamma, \sum_{\sigma \notin \mathscr{C}} \mathfrak{x}_{i 0} \mathrm{t}_{\sigma}=0
$$

for all $i$. But this means exactly that $R(P(\Gamma))=J(\Gamma)$, which is the commutativity of the first diagram. On the other hand, if $\Gamma$ is in $\mathcal{O}_{i r}$, it contains $\Lambda_{\mathscr{H}}$, so that: 


$$
I(S(\Gamma))=\left(\left(\Omega \otimes_{\mathscr{R}} \Lambda\right) \Gamma\left(\Omega \otimes_{\mathscr{R}} \Lambda\right)\right) \cap \mathscr{A}=\left(\Omega \otimes_{\mathscr{R}}(\Lambda \Gamma \Lambda)\right) \cap \mathscr{A}
$$

and the order on the right contains $p(\Gamma)=\Lambda \Gamma \Lambda$. Conversely, an element $\mathfrak{r}$ of $I(S(\Gamma))$ can be written in the form:

$$
\begin{aligned}
\mathfrak{x} & =\sum_{\mathrm{i}} \mathfrak{w}_{\mathrm{i}} \otimes \mathfrak{y}_{\mathrm{i}}=1 \otimes \mathfrak{z} \\
& \left(\mathfrak{w}_{\mathrm{i}} \text {, an } \mathscr{R} \text {-basis of } \Omega ; \mathfrak{y}_{\mathrm{i}} \in \Lambda \Gamma \Lambda=p(\Gamma) ; z \in \mathscr{A}\right) .
\end{aligned}
$$

If $\alpha_{\mathrm{i}}$ are elements of $\mathscr{L}$ such that $1=\sum_{\mathrm{i}} \mathfrak{w}_{\mathrm{i}} \alpha_{\mathrm{i}}$ we obtain at once that $\mathfrak{y}_{\mathrm{i}}=\alpha_{\mathrm{i}} z$ so that $z=\sum_{\mathrm{i}} \mathfrak{w}_{\mathrm{i}} \mathfrak{y}_{\mathrm{i}} \in p(\Gamma)$. This proves the commutativity of the second diagram. The rest follows because of Proposition 2.

CoRollary. Let $\mathscr{J}$ be the inertia group of $\mathscr{E} / \mathscr{F}$ and $\mathscr{E}_{5}=: \mathscr{L}$ the inertia field, and let us assume that the order $\Lambda_{0}$ contains $\Omega=$ $\Lambda_{0 \mathscr{S}}=\Omega_{\mathscr{S}}$ (the inertia ring of the maximal $\mathscr{R}$-order in $\mathscr{E}$ ). Then the lattice $\mathcal{O}$ of orders in $\mathscr{A}$ is isomorphic to the lattice of $\Omega$ orders in $\mathscr{A}_{5}$. The isomorphism preserves hereditary orders. Hence, the study of orders containing the crossed product $\Lambda=\Lambda\left(\mathfrak{f}, \Lambda_{0}, \mathscr{G}\right)$ is reduced to the study of orders containing $\Lambda_{\mathscr{S}}=\Delta\left(\mathfrak{f}, \Lambda_{0}, \mathscr{J}\right), a$ classical crossed product order corresponding to a Galois extension $\mathscr{E} \mid \mathscr{L}$ which is totally ramified. In particular, if $\mathscr{E} / \mathscr{F}$ is an inertial extension and $\Lambda_{0}$ is the maximal $\mathscr{R}$-order in $\mathscr{E}$, then $\Lambda=$ $\Delta\left(\mathfrak{f}, \Lambda_{0}, \mathscr{G}\right)$ is a maximal order in $\mathscr{A}$. [This last statement has been proved by Auslander and Goldman (see Th. A.12 and Prop. 7.1 of The Brauer group of a commutative ring).]

4. Reduction to the wildly remified case. In [2] we introduced in a first approach the idea of a noncommutative crossed product. For the time being, we write below an ad-hoc definition which suffices for the purposes of the present paper.

Let $\mathscr{S}$ be a ring, $\mathscr{B}$ an $\mathscr{S}$-algebra and $\overline{\mathscr{G}}$ a finite group. Given a mapping $\phi: \overline{\mathscr{G}} \longrightarrow \operatorname{Aut}(\mathscr{B})$ such that:

(a) $\phi_{1}=$ ib

(b) $\phi_{o} \phi_{\tau} \phi_{\sigma \tau}^{-1} \in \operatorname{In} \operatorname{Aut}(\mathscr{B})$,

we define the two mappings:

$$
\begin{aligned}
& a: \overline{\mathscr{G}} \times \overline{\mathscr{G}} \longrightarrow \operatorname{In} \operatorname{Aut}(\mathscr{B}) \\
& (\sigma, \tau) \longrightarrow a_{\sigma, \tau} \\
& \mathrm{f}: \overline{\mathscr{G}} \times \overline{\mathscr{G}} \longrightarrow U(\mathscr{B}) \\
& (\sigma, \tau) \longrightarrow \mathrm{f}(\sigma, \tau)
\end{aligned}
$$

by the conditions:

(i ) $\phi_{o} \phi_{\sigma}=a_{\sigma, \tau} \phi_{\sigma \tau}$

(ii) $a_{o, \tau}(\mathfrak{x})=\mathrm{f}(\sigma, \tau) \mathfrak{f f}(\sigma, \tau)^{-1} \quad(\forall \sigma, \tau \in \bar{G}, \forall \mathfrak{x} \in \mathscr{B})$. 
Let $\left\{\mathrm{t}_{o}\right\}(\sigma \in \mathscr{G})$ be any set in $1-1$ correspondence with $\overline{\mathscr{G}}$ and let $\mathscr{A}$ be the free $\mathscr{B}$-module with basis $\left\{\mathrm{t}_{\sigma}\right\}$. Then the rules:

(i ) $\mathfrak{t}_{o} \mathfrak{b}=\phi_{o}(\mathfrak{b}) \mathfrak{t}_{\sigma}$,

(ii) $\mathrm{t}_{\sigma} \mathrm{t}_{\tau}=\mathfrak{f}(\sigma, \tau) \mathrm{t}_{\sigma \tau} \quad(\sigma, \tau \in \overline{\mathscr{G}} ; \mathfrak{b} \in \mathscr{B})$

define a multiplication in $\mathscr{A}$ which makes it into an $\mathscr{S}$-algebra. This algebra will be denoted by $\Delta(\phi, \mathscr{B}, \overline{\mathscr{G}})$.

DEFINITION 3. If $\mathscr{F}$ is any field and $\mathscr{B}$ is a finite dimensional $\mathscr{F}$-algebra, any algebra $\tilde{\mathscr{B}}$ which is isomorphic to one of the form $\Delta(\phi, \mathscr{B}, \overline{\mathscr{G}})$ discussed above, will be called a (noncommutative) crossed product of $\overline{\mathscr{G}}$ over $\mathscr{B}$. If $\Gamma$ is an $\mathscr{R}$-order in $\mathscr{B}$ which is invariant under each of the $\phi_{o}$ 's any order $\widetilde{\Gamma}$ which is isomorphic to $\Delta(\phi, \Gamma, \bar{G})$ will be called a (noncomutative) crossed product of $\overline{\mathscr{G}}$ over $\Gamma$. (For more details see $[2], \S 6$.

The proof of the following result is fairly direct, but rather long. It is written in details in [2].

LEMma 4 (Maschke's theorem). Let $\mathscr{F}$ be any field and $\mathfrak{p}$ its characteristic and let $\tilde{\mathscr{B}}=\Delta(\phi, \mathscr{B}, \overline{\mathscr{G}})$ be a crossed product $\mathscr{F}$ algebra. Then, if $\mathfrak{p}$ does not divide the order of $\overline{\mathscr{G}}$, we have:

$\mathscr{B}$ is semisimple $\widetilde{\mathscr{B}}$ is semisimple;

$\mathscr{B}$ is separable $\Longrightarrow \mathscr{\mathscr { B }}$ is separable.

For the remaining of this section we go back to the notations of $\S 3$. The situation of Proposition 3 provides us with an example of noncommutative crossed product algebra and an example of noncommutative crossed product order, which is actually the main justification for the introduction of this idea in the present paper.

Let us make $\mathscr{A}_{\mathscr{H}}$ play the role of $\mathscr{B}$, and $\mathscr{G} / \mathscr{H}$ the role of $\overline{\mathscr{G}}$. Let us choose a set of representatives of $\mathscr{G}$ in $\overline{\mathscr{G}}$, and let, in general, $\sigma$ be the representative of the coset $\bar{\sigma} \in \overline{\mathscr{G}}$. Let $\phi$ be the mapping which sends each $\bar{\sigma} \in \overline{\mathscr{G}}$ to the automorphism of $\mathscr{A}_{\mathscr{C}}$ which is conjugation by $\mathrm{t}_{\sigma}$. Then $\mathscr{A}$ is a crossed product $\Delta(\phi, \mathscr{A} \mathscr{\mathscr { C }}, \mathscr{G} / \mathscr{H})$. In a similar way, $\Lambda$ is a crossed product order: $\Lambda=\Delta\left(\phi, \Lambda_{\mathscr{C}}, \mathscr{G} \mid \mathscr{H}\right)$, and, more generally, for each $\Gamma \in \mathcal{O}_{\mathscr{K}}^{\prime}, p(\Gamma)=\Lambda \Gamma \Lambda=\Delta(\phi, \Gamma, \mathscr{G} \mid \mathscr{H})$.

Proposition 4. If the characteristic of $\mathscr{F}$ does not divide the order of $\overline{\mathscr{G}}=\mathscr{G} / \mathscr{H}$, we have:

1. For each $\Gamma \in \mathcal{O}_{\mathscr{C}}^{\prime}, \operatorname{rad}(p(\Gamma))=\operatorname{rad}(\Gamma) \cdot p(\Gamma)$;

2. $\Gamma \in \mathcal{O}_{\mathscr{H}}^{\prime}$ is hereditary if and only if $p(\Gamma)$ is hereditary;

3. $\forall \Gamma \in \mathcal{O}$, if $J(\Gamma)$ is hereditary, then $\Gamma$ is hereditary. 
Note. This proposition applies in the case that $\mathscr{E} / \mathscr{F}$ is totally ramified when $\mathscr{H}=\mathscr{R}$, the first ramification group. In this case, $\mathscr{A}_{\mathscr{C}}=\mathscr{A}_{\mathscr{R}_{1}}=\Lambda\left(\mathfrak{f} \mid \mathscr{R}_{1} \times \mathscr{R}_{1}, \mathscr{E}, \mathscr{R}_{1}\right)$ is a $\mathscr{V}$-algebra, where $\mathscr{V}$ is the ramification field of $\mathscr{E} / \mathscr{F}$. The extension $\mathscr{E} / \mathscr{V}$ is wildly namified. Thus, this proposition provides a reduction for our study to the wildly ramified case.

Proof. 1. Let $\mathscr{N}$ be the radical of $\Gamma$ and let

$$
\mathscr{N}^{\prime}=\Delta(\phi, \mathscr{N}, \mathscr{G} \mid \mathscr{C}) \text {. }
$$

Then $\mathscr{N}^{\prime}$ is a two-sided ideal of $p(\Gamma)$ and, by Nakayama's lemma, $\mathscr{N}^{\prime} \subset \operatorname{rad}(p(\Gamma))$. On the other hand,

$$
p(\Gamma) / \mathscr{N}^{\prime}=\Delta(\phi, \Gamma, \mathscr{G} \mid \mathscr{H}) / \Delta(\phi, \mathscr{N}, \mathscr{G} \mid \mathscr{H}) \cong \Delta(\bar{\phi}, \Gamma / \mathscr{N}, \mathscr{G} \mid \mathscr{H}),
$$

where, for each $\bar{\sigma} \in G / H, \bar{\phi}_{\bar{\sigma}}$ is the passage to the quotient $\Gamma / \mathscr{N}$ of the automorphism $\phi_{\bar{\sigma}}$ of $\Gamma$. Then, by Maschke's theorem, $p(\Gamma) / \mathscr{N}^{\prime}$ is semisimple, whence $\operatorname{rad}(p(\Gamma))=\mathscr{N}^{\prime}=\mathscr{N} \cdot p(\Gamma)=: p(\mathscr{N})$.

2. Let $\Gamma \in \mathcal{O}_{\mathscr{C}}^{\prime}$ be hereditary. Then there are elements $x_{i} \in \mathscr{N}$, $\mathfrak{x}_{i}^{\prime} \in \mathscr{N}^{-1}$ such that $\sum \mathfrak{x}_{i}^{\prime} \mathfrak{x}_{i}=1$. It follows from 1 that $\mathfrak{x}_{i} \in p(\mathscr{N})$, $\mathfrak{x}_{i}^{\prime} \in p(\mathscr{N})^{-1}$, so that $p(\Gamma)$ is hereditary.

Now let $p(\Gamma)$ be hereditary and let $\left\{\hat{x}_{i}\right\}$ be an $\mathscr{R}$-basis of $p(\mathscr{N})$ and $\mathfrak{x}_{i}^{\prime}$ be elements of $p(\mathscr{N})^{-1}$ such that $\sum \mathfrak{x}_{i}^{\prime} \mathfrak{x}_{i}=1$. We can write each $\mathfrak{r}_{\mathrm{i}}$ in the form $\mathfrak{y}_{\mathrm{i}} \mathrm{t}_{\bar{\sigma}}$, where $\left\{\mathfrak{y}_{\mathrm{i}}\right\}$ is an $\mathscr{R}$-basis of $\mathscr{N}$. If $\mathfrak{x}_{\mathrm{i}}$ happens to be, in this notation, $\mathfrak{y}_{\mathrm{i}} \mathrm{t}_{\bar{\sigma}}$ we denote it $\mathfrak{x}_{\mathrm{i}, \bar{\sigma}}$ and $\mathfrak{x}_{\mathrm{i}, \bar{\sigma}}^{\prime}$ the corresponding $\mathfrak{x}_{i}^{\prime}$. Hence, we can write:

$$
\mathfrak{X}_{\mathrm{i}, \bar{\sigma}}^{\prime}=\sum_{\bar{\tau} \in \mathscr{S} \mid \mathscr{\mathscr { C }}} \mathfrak{b}_{\mathrm{i}, \bar{\sigma}, \bar{\tau}} \mathrm{t}_{\bar{\tau}} . \quad\left(\mathfrak{b}_{\mathrm{i}, \bar{\sigma}, \bar{\tau}} \in \mathscr{C}_{\mathscr{C}}\right) .
$$

It follows easily that each $\mathfrak{b}_{j, \bar{\sigma}, \bar{\tau}}$ belongs to $\mathscr{N}^{-1}$ and since

$$
\sum_{\mathrm{i}, \bar{\sigma}, \bar{\tau}} \mathfrak{b}_{\overline{\mathrm{i}}, \bar{\sigma}, \overline{\mathrm{t}}, \mathrm{t}} \mathfrak{\mathfrak { y }}_{\mathrm{i}} \mathbf{t}_{\bar{\sigma}}=1
$$

which implies

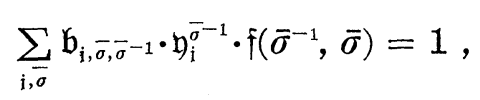

we have that $\Gamma$ is hereditary.

3. We have $p(J(\Gamma)) \subset \Gamma$. Hence, if $J(\Gamma)$ is hereditary, $p(J(\Gamma))$ is hereditary, by 2 , and $\Gamma$ is hereditary because it contains the latter.

Definition 4. Let $\tilde{\Lambda}=\Lambda(\phi, \Lambda, \overline{\mathscr{G}})$ be a noncommutative crossed product in a separable $\mathscr{F}$-algebra $\tilde{\mathscr{A}}=\Delta(\phi, \mathscr{A}, \overline{\mathscr{G}})$, where $\mathscr{A}$ is a central simple $\mathscr{F}$-algebra. Let $\mathscr{F}(\Lambda)$ be the center of $\Lambda$. Then:

(1) The inertia group, $\mathscr{F}$, of $\tilde{\Lambda}$ over $\Lambda$ is: 


$$
\mathscr{F}=\left\{\sigma \in \overline{\mathscr{G}} / \forall \mathfrak{x} \in \mathscr{F}(\Lambda), \phi_{\sigma}(\mathfrak{r}) \equiv \mathfrak{r} \bmod (\operatorname{rad}(\Lambda))\right\}
$$

( $\mathscr{F}$ is a normal subgroup of $\overline{\mathscr{G}}$ );

(2) $\tilde{A}$ is tamely ramified over $\Lambda$ if

(i) $\mathscr{Z}(\Lambda / \operatorname{rad}(\Lambda))$ is a field, an algebraic, separable extension of $\overline{\mathscr{F}}$ and

(ii) The characteristic, $\mathfrak{p}$, of $\overline{\mathscr{F}}$ does not divide the order of $\mathscr{F}$

THEOREM 2. With the notations of Definition 4, if $\tilde{\Lambda}$ is tamely ramified over $\Lambda$ and if $\Lambda$ is hereditary, then $\tilde{\Lambda}$ is hereditary and $\operatorname{rad}(\widetilde{\Lambda})=\operatorname{rad}(\Lambda) \cdot \widetilde{\Lambda}$. [Note: In fact, for the proof of this theorem we do not make use of the separability of $\left.\mathscr{Z}(\Lambda / \operatorname{rad}(\Lambda)) / \bar{F}_{\text {.] }}\right]$

Proof. It is enough to prove the latter statement (cf. the proof of Proposition 4). As in Proposition 4, we can write $\bar{\Gamma}=\tilde{\Lambda} / \operatorname{rad}(\Lambda) \tilde{\Lambda}$ in the form $\Delta(\bar{\phi}, \Lambda / \operatorname{rad}(\Lambda), \overline{\mathscr{G}})$, and it would be enough to show that this algebra is semisimple. Let $\bar{\Gamma}_{0}=\Delta(\bar{\phi}, \Lambda / \operatorname{rad}(\Lambda), \mathscr{F})$. Then $\bar{\Gamma}$ is in an obvious way a noncommutative crossed product of $\mathscr{G} / \mathscr{I}$ over $\bar{\Gamma}_{0}$. By Maschke's theorem, $\bar{\Gamma}$ is semisimple. Also, it is clear that $\operatorname{rad}(\bar{\Gamma}) \cap \bar{\Gamma}_{0} \subset \operatorname{rad}\left(\bar{\Gamma}_{0}\right)=0$. By contradiction, we assume that $\operatorname{rad}(\bar{\Gamma}) \neq$ 0 , and take $\delta \neq 0, \delta \in \operatorname{rad}(\bar{\Gamma})$. We write $\delta$ as a linear combination:

$$
\delta=\sum \delta_{i} t_{\sigma_{1}} \quad\left(\delta_{i} \in \bar{\Gamma}_{0}\right)
$$

wite a minimal set of nonzero coefficients and we can assume, w.l.o.g., that one of the $t_{\sigma_{1}}$ is $t_{\sigma_{1}}=1$. Also, since $\delta \neq \mathcal{O}$, the number of coefficients is at least two. Let $\mathfrak{x}$ be an element of $\mathscr{z}(\Lambda)$ such that, for some index $i$ which we keep fixed thereafter,

$$
\dot{\phi}_{\sigma_{\mathrm{i}}}(\mathfrak{x}) \not \equiv \mathfrak{x} \bmod (\operatorname{rad}(\Lambda)),
$$

so that if $\overline{\mathrm{x}}$ is its class in $\bar{\Gamma}_{0}, \bar{\phi}_{\sigma_{\mathrm{i}}}(\overline{\mathrm{x}}) \neq \overline{\mathrm{x}}$. We have:

$$
\begin{aligned}
& \overline{\mathfrak{x}} \cdot \delta=\sum \overline{\mathfrak{x}} \delta_{\mathrm{i}} \mathrm{t}_{\sigma_{\mathrm{i}}}=\sum \delta_{\mathrm{i}} \overline{\mathrm{r}} t_{\sigma_{\mathrm{i}}} \\
& \delta \cdot \bar{\phi}_{\sigma_{\mathrm{i}}}^{-1}(\overline{\mathfrak{x}})=\sum \delta_{\mathrm{i}} \bar{\phi}_{\sigma_{\mathrm{i}}} \bar{\phi}_{\sigma_{\mathrm{i}}}^{-1}(\overline{\mathfrak{x}}) \mathrm{t}_{\sigma_{\mathrm{i}}}
\end{aligned}
$$

By the assumption of minimality, it follows that $\bar{x} \delta-\delta \bar{\phi}_{\sigma_{\mathrm{i}}}^{-1}(\overline{\mathrm{x}})=0$, which implies $\delta_{1} \cdot\left(\overline{\mathfrak{x}}-\bar{\phi}_{\sigma_{\mathrm{i}}}^{-1}(\overline{\mathfrak{x}})\right)=0$, a contradiction.

CoRollaRy If $\Gamma \in \mathcal{O}_{\mathscr{H}}^{\prime}$ is a maximal order and $\mathfrak{p} \nmid[\mathscr{J}: \mathscr{H}]$, where $\mathscr{J} / \mathscr{H}$ is the inertia group of $p(\Gamma)$ over $\Gamma$, then $p(\Gamma)$ is hereditary.

Proof. We know that $\mathscr{A}_{\mathscr{C}}$ is simple and if $\Gamma$ is maximal, $\Gamma / \mathrm{rad}(\Gamma)$ is a simple algebra whose center is a field, so that the proof of Theorem 2 applies to this case. 
Note. This paper has been written under the assumption that for Galois (field) extensions $\mathscr{E} / \mathscr{F}$ the residue class field extension $\overline{\mathscr{E}} \mid \overline{\mathscr{F}}$ is separable (cf. Cor. to Prop. 3, Prop. 4). This assumption is convenient for our purposes, but is not really necessary.

\section{REFERENCES}

1. M. Harada, Some criteria for hereditarity of crossed product orders, Osaka J. Math., 1 (1964), 69-80.

2. H. Merklen, On crossed product orders, $\mathrm{Ph}$. D. Thesis, The Ohio State University, Columbus, Ohio (1972).

3. K. W. Roggenkamp and V. Huber-Dyson, Lattices Over Orders I, Springer-Verlag (1970).

4. K. W. Roggenkamp, Lattices Over Orders II, Springer-Verlag (1970).

5. S. Williamson, Crossed products and hereditary orders, Nagoya Math. J., 23 (1963), $103-120$.

6. S. Williamson, $\pi$-principal hereditary orders, Nagoya Math. J., 32 (1968), 41-65.

Received November 1, 1973 and in revised form May 6, 1975. The main results in this paper are partially contained in the author's Ph.D. thesis at The Ohio State University, Columbus, Ohio, work which was supervised by Prof. H. Zassenhaus. The publication of this paper was financed by the Fundaçãd de Amparo À Pesquisa do Estado de São Paulo, Brazil.

Instituto De Matemática e Estatistica

UNIVERSidADE DE São PAULO

SÃo PaUlo, BRazil 


\section{PACIFIC JOURNAL OF MATHEMATICS}

\section{EDITORS}

RICHARD ARENS (Managing Editor)

University of California

Los Angeles, CA 90024

Charles W. Curtis

University of Oregon

Eugene, OR 97403

C. C. Moore

University of California

Berkeley, CA 94720

\section{J. DugundJI}

Department of Mathematics

University of Southern California

Los Angeles, CA 90007

R. FInN and J. Milgram

Stanford University

Stanford, CA 94305

\section{ASSOCIATE EDITORS}
E. F. BECKENBACH
B. H. NeumanN
F. WOLF
K. YoSHIDA

\section{SUPPORTING INSTITUTIONS}

\author{
UNIVERSITY OF BRITISH COLUMBIA \\ CALIFORNIA INSTITUTE OF TECHNOLOGY \\ UNIVERSITY OF CALIFORNIA \\ MONTANA STATE UNIVERSITY \\ UNIVERSITY OF NEVADA, RENO \\ NEW MEXICO STATE UNIVERSITY \\ OREGON STATE UNIVERSITY \\ UNIVERSITY OF OREGON
}

\author{
UNIVERSITY OF SOUTHERN CALIFORNIA \\ STANFORD UNIVERSITY \\ UNIVERSITY OF HAWAII \\ UNIVERSITY OF TOKYO \\ UNIVERSITY OF UTAH \\ WASHINGTON STATE UNIVERSITY \\ UNIVERSITY OF WASHINGTON
}

The Supporting Institutions listed above contribute to the cost of publication of this Journal, but they are not owners or publishers and have no responsibility for its content or policies.

Mathematical papers intended for publication in the Pacific Journal of Mathematics should be in typed form or offset-reproduced, (not dittoed), double spaced with large margins. Please do not use built up fractions in the text of the manuscript. However, you may use them in the displayed equations. Underline Greek letters in red, German in green, and script in blue. The first paragraph or two must be capable of being used separately as a synopsis of the entire paper. Items of the bibliography should not be cited there unless absolutely necessary, in which case they must be identified by author and journal, rather than by item number. Manuscripts, in triplicate, may be sent to any one of the editors. Please classify according to the scheme of Math. Reviews, Index to Vol. 39. All other communications should be addressed to the managing editor, or Elaine Barth, University of California, Los Angeles, California, 90024.

50 reprints to each author are provided free for each article, only if page charges have been substantially paid. Additional copies may be obtained at cost in multiples of 50 .

The Pacific Journal of Mathematics is issued monthly as of January 1966. Regular subscription rate: $\$ 72.00$ a year (6 Vols., 12 issues). Special rate: $\$ 36.00$ a year to individual members of supporting institutions.

Subscriptions, orders for numbers issued in the last three calendar years, and changes of address should be sent to Pacific Journal of Mathematics, 103 Highland Boulevard, Berkeley, California, 94708. Older back numbers obtainable from Kraus Periodicals Co., Route 100, Millwood, NY 10546.

PUBLISHED BY PACIFIC JOURNAL OF MATHEMATICS, A NON-PROFIT CORPORATION

Printed at Kokusai Bunken Insatsusha (International Academic Printing Co., Ltd.). 8-8, 3-chome, Takadanobaba, Shinjuku-ku, Tokyo 160, Japan.

Copyright (C) 1978 by Pacific Journal of Mathematics

Manufactured and first issued in Japan 


\section{Pacific Journal of Mathematics

Vol. 74, No. $2 \quad$ June, 1978

Aharon Atzmon, Spectral synthesis in some spaces of bounded continuous

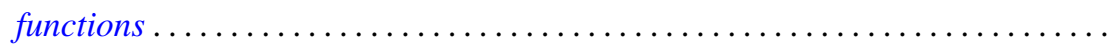

Karl Egil Aubert and Isidor Fleischer, Tensor products of ideal systems and their modules.............................................

Richard F. Basener, Several dimensional properties of the spectrum of a uniform

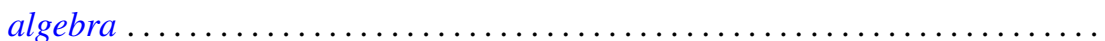

R. H. Bing and Michael Peter Starbird, Super triangulations ............. 307

Andrew Carson, Coherent polynomial rings over regular rings of finite index .....

Robert M. DeVos and Frederick W. Hartmann, Sequences of bounded summability domains .................................................

George Grätzer and R. Padmanabhan, Symmetric difference in abelian groups ....

Robert L. Griess, Jr., A remark about groups of characteristic 2-type and

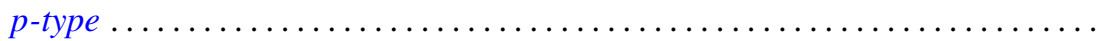

Emil Grosswald and F. J. Schnitzer, A class of modified $\zeta$ and L-functions........

Jutta Hausen and Johnny Albert Johnson, Ideals and radicals of some

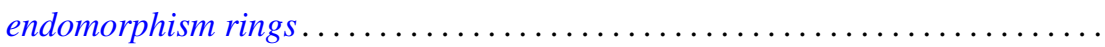

Jean Ann Larson, A solution for scattered order types of a problem of Hagendorf ............................................

Peter A. McCoy, Extremal properties of real biaxially symmetric potentials in $E^{2(\alpha+\beta+2)}$

Héctor Alfredo Merklen, Hereditary crossed product orders .

Hal G. Moore and Adil Mohamed Yaqub, Equational definability of addition in certain rings...

Robert Laurens Moore, Reductivity in $C^{*}$-algebras and essentially reductive operators. . .

Joseph Alvin Neisendorfer, Lie algebras, coalgebras and rational homotopy theory for nilpotent spaces...

William Raymond Nico, Bounded monoids

Richard Paul Osborne, Simplifying spines of 3-manifolds ...

Richard Paul Osborne, The simplest closed 3-manifolds. With an appendix by Osborne and J. Yelle.

Clayton Collier Sherman, The $K$-theory of an equicharacteristic discrete valuation ring injects into the $K$-theory of its field of quotients.... .

Mitchell Herbert Taibleson, The failure of even conjugate characterizations of $H^{1}$

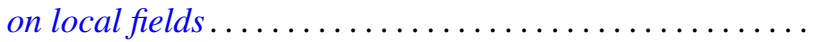

Keti Tenenblat, On characteristic hypersurfaces of submanifolds in Euclidean space ...................................

Jeffrey L. Tollefson, Involutions of Seifert fiber spaces..........

Joel Larry Weiner, An inequality involving the length, curvature, and torsions of a curve in Euclidean $n$-space .......................

Neyamat Zaheer, On generalized polars of the product of abstract homogeneous polynomials.... 